\title{
T-eigenfaces selection for false face reduction
}

\author{
Jing-Wein Wang ${ }^{\mathrm{a})}$ \\ Institute of Photonics and Communications, National Kaohsiung University of \\ Applied Sciences, \\ 415 Chien Kung Road, Kaohsiung, Taiwan 807, R.O.C. \\ a)jwwang@cc.kuas.edu.tw
}

Abstract: We have developed a false face reduction algorithm dedicated for face detection in an unconstrained environment, based on the discriminative facial T-shape region constructed by human eyes, nose, and mouth. The algorithm started with a detected face, through lighting compensation, normalization, facial T-shape region extraction, and eigenface selection using genetic algorithms to classify face/false face robustly. Results of face detection with and without the proposed false face reducer show substantial improvements on precision rate at a little loss of recall rate.

Keywords: false face reduction, face detection, facial T-shape region, genetic algorithms

Classification: Science and engineering for electronics

\section{References}

[1] M.-H. Yang, D. Kriegman, and N. Ahuja, "Detecting faces in images: a survey," IEEE Trans. Pattern Anal. Mach. Intell., vol. 24, pp. 34-58, 2002.

[2] P. Viola and M. Jones, "Robust Real-time Face Detection," International Journal of Computer Vision, vol. 57, pp. 137-154, 2004.

[3] J. Dios, "Skin Color and Feature-Based Segmentation for Face Localization," Optical Engineering, vol. 46, pp. 037007-1-037007-6, 2007.

[4] [online] http://www.ee.surrey.ac.uk/CVSSP/banca/, The BANCA database.

[5] J.-W. Wang, "Precise Face Segmentation for Recognition," IEEE International Conf. on Image Processing (ICIP2006), Atlanta, USA, pp. 2045-2048, Oct. 8-11, 2006.

[6] [online] http://www.bioid.com/downloads/facedb/index.php, BioID face detection database.

[7] [online] http://www.kasrl.org/jaffe.html, Japanese Female Facial Expression (JAFFE) database.

[8] [online] http://www.abcnews.go.com/, ABC news photos.

[9] [online] http://www.idiap.ch/ ${ }^{\sim}$ marcel/cv.php, IDIAP face tracker.

[10] [online] http://www.betaface.com/, Betaface online detector. 


\section{Introduction}

Face detection has been receiving extensive attention with increasing demands in locating faces correctly [1]. Usually, face detection is achieved by training artificial neural networks and measuring distances between training sets in order to detect candidates that might indicate a human face [2]. Another popular method for doing face detection is by using skin color and anthropometry information [3]. In spite of many face detectors have used face verification process to reduce the number of false alarms, one of difficult problems in face detection is that the detecting performance is significantly influenced by many variables in locating faces, e.g., lighting, pose, facial expression, glasses, and cluttered backgrounds, which may vary depending on environmental factors of face image acquisition. It has been determined that false-positive classifications are frequently encountered in face detection. Since the false acceptance of face resulted from an incorrect declaration will cause more risk than the false rejection of face, it has been a motor for the development of how to reduce the false detections without decreasing the face detection rate, but hardly any method was reported to address it. In this paper, we present a new method for reducing false faces using T-eigenfaces, short for eigenfaces based on facial T-shape region constructed by eyes, nose, and mouth. There are two stages in our algorithm: a feature extraction stage and a classification stage. In the first stage, T-eigenfaces are extracted from a normalized detected face through lighting compensation, and in the second stage, genetic algorithms (GAs) are used to select the most discriminative Teigenface subset with the proposed fitness function and the Mahalanobis classifier via leave-one-out cross-validation, to which the subset has the largest fitness value.

\section{T-eigenface Extraction and Selection}

The algorithm's details of T-eigenfaces extraction and selection as illustrated in Fig. 1 (a) are described as follows:

Step 1: Normalize the detected face image to resize to $64 \times 64$ pixels, which is considered as a suitable medium size for presumed faces ranging from the smallest $24 \times 24$ pixels to the largest $160 \times 160$ pixels.

Step 2: Perform lighting compensation on the normalized image to alleviate lighting variation presented at face area in the following ways. The image $f$ is first scaled with a ratio of the reference value 120 to the mean gray value $\mu$ so as to redistribute grey values and confine the dynamic range of exposure to a small amount.

$$
f^{\prime}(x, y)=f(x, y) \times \frac{120}{\mu} .
$$

Then we take a localized tuning for the scaled pixels with gray values larger than or equal to 120 and smaller than 120 , respectively.

$$
\begin{aligned}
& f^{\prime \prime}(x, y)=\left(f^{\prime}(x, y)-\mu_{u}\right) / \sigma_{u}+\mu_{u}, \forall \quad f^{\prime}(x, y) \geq 120 \\
& f^{\prime \prime}(x, y)=\mu_{l}-\left(\mu_{l}-f^{\prime}(x, y)\right) / \sigma_{l}, \forall \quad f^{\prime}(x, y)<120
\end{aligned}
$$




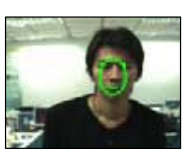

Detected

elliptic face input

$\checkmark$

Normalization \& Lighting compensation
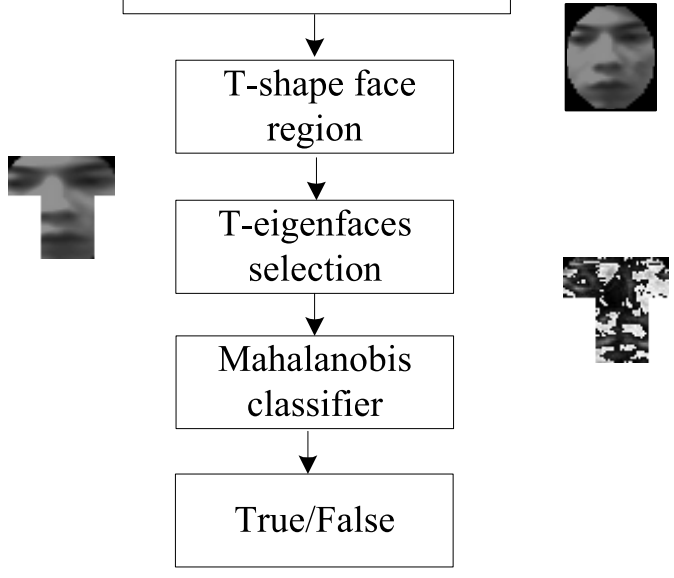

(a)
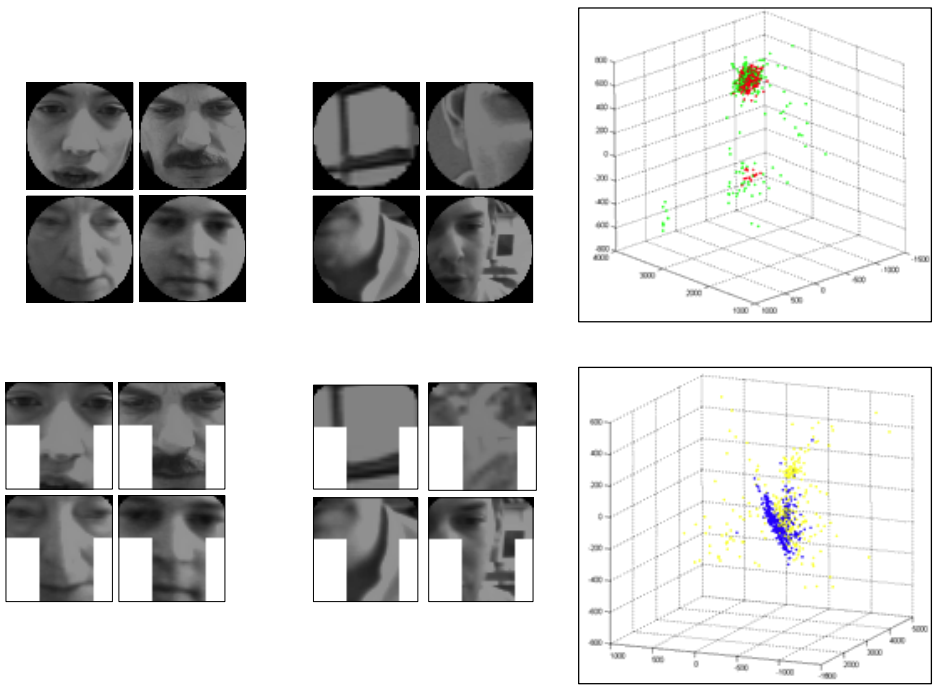

(b)

Fig. 1. (a) Proposed false face reducer, (b) Clustering of faces and false faces by using the leading three eigenfaces. Elliptic face: face in red color and false face in green color; T-shape face: face in blue color and false face in yellow color.

where $\mu_{u}$ and $\sigma_{u}$ are the mean and the variance of pixel values larger than or equal to 120 , respectively, and $\mu_{l}$ and $\sigma_{l}$ are accordingly defined for pixel values smaller than 120, respectively.

Step 3: Segment the T-shape region of the normalized and lightingcompensated face out to take away cheek areas.

Step 4: Extract T-eigenfaces by using principal component analysis (PCA) technique, which is based on extracting the axes on which the data shows 
the highest variability. To locate the discriminatory subset, which explains as much of the discriminatory power in the data as possible with as few of these as possible, the fitness function is proposed below to genetically select a subset that has the largest fitness value by using the Mahalanobis classifier through leave-one-out cross-validation.

$$
\text { fitness }=\varsigma \cdot\left(\frac{\alpha}{\beta}\right) \cdot\left(1-\frac{\vartheta_{s}}{\vartheta_{t}}\right),
$$

where $\alpha$ is the number of accurate acceptance and rejection, $\beta$ is the number of false acceptance and rejection, $\vartheta_{s}$ is number of the selected T-eigenfaces, $\vartheta_{t}$ is the total number of T-eigenfaces, and $\varsigma$ is a weighting factor which has been set as 2.0 in the experiment. The basic idea in GAs is to maintain a population of knowledge structures (chromosomes) that represents a pool of possible solutions of the optimization problem. With our method, a binary mask vector associated with T-eigenfaces for candidate solutions is encoded on the GA chromosome. If the $i$ th bit of this vector equals 1 , the $i$ th variable is included in the classifier in classification. Otherwise, the corresponding variable is not considered for classification. Each subset of T-eigenfaces is evaluated according to its evaluated fitness on a set of testing data using the Mahalanobis classifier. As in natural evolution, survival of the fittest is the main strategy. The selected chromosomes are altered using single-point crossover operator to form new fit chromosomes from fit parents. A mutation operator with a small probability less than the crossover probability, as in natural evolution, serves as a secondary search operator that ensures the reach ability of all points in the search space. This process continues until either an absolute fittest chromosome is detected in the population or a predetermined maximum number of generations is reached. Then, the best string with the highest fitness value at the last generation is preserved for further use in the classification phase, where a detected face is matched against the selected T-eigenface subset and the true/false decision through the Mahalanobis's threshold is taken as the classification result. In the end, the recall rate is determined as the ratio of the number of successful detections to labeled faces in the dataset, while the precision rate is calculated as the ratio of the number of successful detections to the number of detected windows.

\section{Experimental Results and Discussions}

One of popular databases could provide the challenges that face detection algorithms can encounter in real applications: such as poor image quality, presence of multiple faces, and faces with upright and near-frontal views is from BANCA [4]. The database with 6240 color images of faces has three parts, i.e. controlled, degraded, and adverse recording sessions. Our training set contains 4,537 BANCA faces and 1,000 false faces that were extracted by our elliptic face detector [5] from various sources including internet and pictures taken by ourselves. The false face reducer is tested on the face images 
from the BANCA, BioID [6], JAFFE [7], ABC news photos [8], and our pictures with 65 faces that differ from the false face reducer's training datasets. In order to analyze the clustering performance of faces and false faces in the cases of elliptic and T-shape face segmentations, we use the leading three eigenfaces derived from PCA to examine the capability of collecting similar objects into groups. With face and false face 400 samples each from the training set, we can see from Fig. 1 (b) that the result from T-eigenfaces is better than the one from elliptic-eigenfaces. Next, we report an investigation of T-eigenfaces designed to improve the performance of the face detection method by focusing on the characteristics of the false face reducer. The reported results for the GA evolutionary classification tasks have the following parameter settings: population size 20, number of generation 2000, and the probability of crossover 0.5 . A mutation probability value starts with a value of 0.1 and then varied as a step function of the number of iterations until it reaches a value of 0.001 . Moreover, we set 300 T-eigenfces taken from the leading ones that experimentally showed containing enough information for face reconstruction for the GA selection task. After experimentation, we selected 116 T-eigenfaces with the Mahalanobis threshold value 72 .

The experiments were conducted to evaluate the performance of face detection with and without false face reducer in terms of recall and precision rates. Fig. 2 shows example results obtained by our elliptic face detector with the proposed false face reducer on the test sets, which are composed of various sources. From the examples presented in Figs. 2 (a)-2(c), the false faces were successfully removed without error. In the pictures of the examples presented in Fig. 2(d), on the other hand, it can be observed that there are two misclassification cases encountered. In the left-hand case of Fig. 2 (d), a face was misclassified as false leading to deterioration in detection rate. As displayed in the right-hand case of Fig. 2 (d), the false face located at the helmet of the second solider from left side is successfully removed, while the other one on building was passed true/false examination and still existed. Fig. 2 (e) shows the results of our pictures and we observed that although false faces presented in both pictures were mostly removed, but leaved a few unaltered. The performance of experiments is detailed in Table 1. From the experimental results, our method can reduce false detections by up to $11 \%$ only at a cost of $4.5 \%$ detection rate for $\mathrm{ABC}$ dataset. In terms of speed, the execution time of the proposed false face reducer is operating at an average processing time less than 0.4 sec per subject on an AMD64 Athlon PC. Note that, as presented in Fig. 2 (f), in addition to the effectiveness connected with our face detector, the algorithm is able to examine the detected windows output from the other detector, e.g., IDIAP [9] which just runs on live video streaming, as well, and have a good performance. Meanwhile, the Betaface online detector [10] is further adopted to show how the system works. To make a comparison with a common face detector, on the other hand, the experimental results of Betafce detector on all datasets by submitting images to website for trials are also tabulated in Table 1. As can be observed, our elliptic detector with false face reducer outperforms than the Betaface 

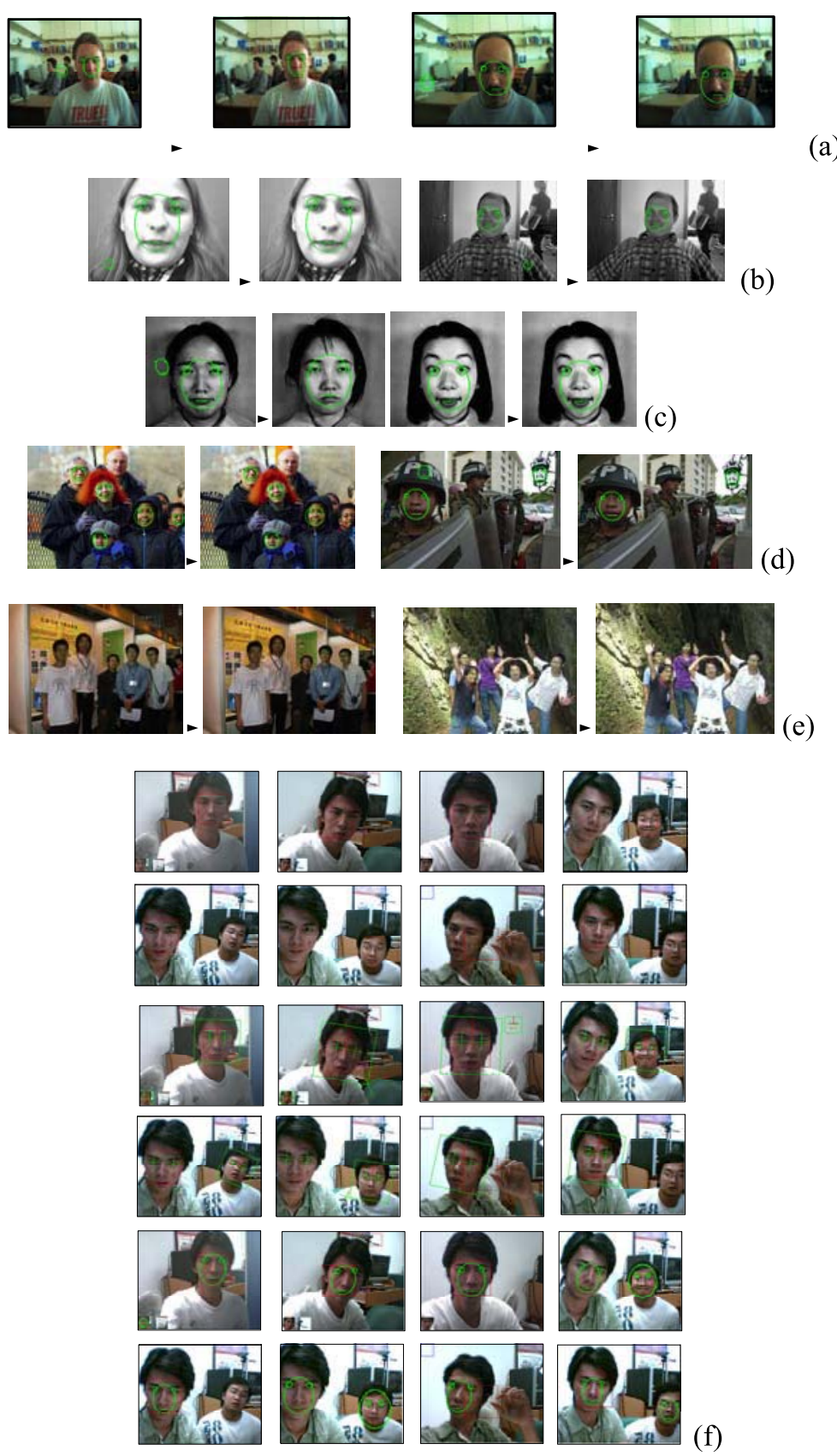

Fig. 2. Face detection examples by using the elliptic face detector our previous algorithm with the proposed false face reducer for various datasets: (a) BANCA, (b) BioID, (c) JAFFE, (d) ABC, (e) Our pictures; Examples of lab scenario shown for comparison with the related works: (f) IDIAP detection framed in red rectangular, Betaface detection framed in green rectangular, and our elliptic detector with false face reducer framed in green ellipse. 
Table I. Performance of the elliptic face detector without/with false face reducer and comparison with the Betaface online detector.

\begin{tabular}{|c|c|c|c|c|}
\hline Dataset & $\begin{array}{c}\text { Elliptic detector/ } \\
\text { Betaface online } \\
\text { detector }\end{array}$ & Recall (\%) & Precision (\%) & $\begin{array}{c}\text { no. of } \\
\text { false } \\
\text { faces }\end{array}$ \\
\hline BANCA & Without & $97.67(1429 / 1463)$ & $86.81(1429 / 1646)$ & 217 \\
\hline & With & $95.89(1403 / 1463)$ & $97.97(1403 / 1432)$ & 29 \\
\hline & Betaface & $94.0(94 / 100)$ & $91.26(94 / 103)$ & 9 \\
\hline BioID & Without & $94.93(1387 / 1461)$ & $89.36(1387 / 1552)$ & 165 \\
\hline & With & $88.09(1287 / 1461)$ & $98.84(1287 / 1302)$ & 15 \\
\hline & Betaface & $90.0(90 / 100)$ & $94.74(90 / 95)$ & 5 \\
\hline JAFFE & Without & $100(213 / 213)$ & $99.53(213 / 214)$ & 1 \\
\hline & With & $100(213 / 213)$ & $100(213 / 213)$ & 0 \\
\hline & Betaface & $99.0(99 / 100)$ & $100(99 / 99)$ & 0 \\
\hline ABC & Without & $91.65(3094 / 3376)$ & $85.23(3094 / 3369)$ & 254 \\
\hline & With & $87.18(2952 / 3376)$ & $96.31(2952 / 3065)$ & 113 \\
\hline & Betaface & $89.0(89 / 100)$ & $89.0(89 / 100)$ & 11 \\
\hline Ours & Without & $84.61(55 / 65)$ & $82.09(55 / 67)$ & 12 \\
\hline & With & $81.54(53 / 65)$ & $92.98(53 / 57)$ & 4 \\
\hline & Betaface & $83.08(54 / 65)$ & $87.1(54 / 62)$ & 8 \\
\hline
\end{tabular}

detector overall, particularly in precision rate.

\section{Conclusion}

We have presented in this paper a framework for false face reduction based on the selected T-eigenfaces yielded by GAs, without making any assumption concerning the contents of the face pattern to analyze. The robustness of our system to varying poses and facial expressions as well as lighting variations was evaluated using various datasets including a real set of our live images. As demonstrated in the results, another merit of our algorithm is that any face detector can be cascaded by our false face reducer to remove false faces by providing their detected faces only.

\section{Acknowledgments}

NSC project no. 96-2221-E-151-051 is acknowledged. 